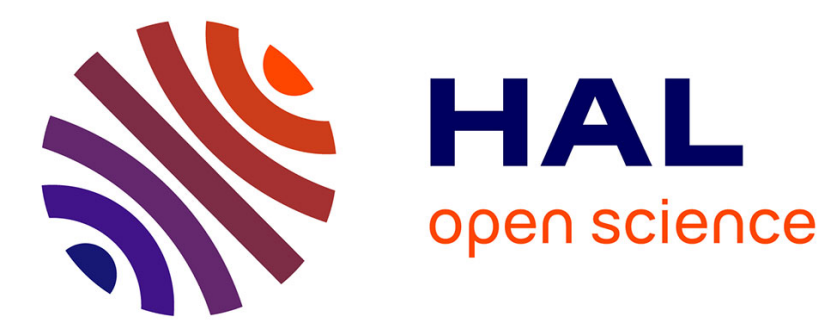

\title{
Magnon band structure of periodic composites
}

Jerome O. Vasseur, L. Dobrzynski, Bahram Djafari-Rouhani, H. Puszkarski

\section{To cite this version:}

Jerome O. Vasseur, L. Dobrzynski, Bahram Djafari-Rouhani, H. Puszkarski. Magnon band structure of periodic composites. Physical Review B, 1996, 54 (2), pp.1043-1049. 10.1103/PhysRevB.54.1043 . hal-03301165

\section{HAL Id: hal-03301165 \\ https://hal.science/hal-03301165}

Submitted on 27 Jul 2021

HAL is a multi-disciplinary open access archive for the deposit and dissemination of scientific research documents, whether they are published or not. The documents may come from teaching and research institutions in France or abroad, or from public or private research centers.
L'archive ouverte pluridisciplinaire HAL, est destinée au dépôt et à la diffusion de documents scientifiques de niveau recherche, publiés ou non, émanant des établissements d'enseignement et de recherche français ou étrangers, des laboratoires publics ou privés. 


\title{
Magnon band structure of periodic composites
}

\author{
J. O. Vasseur,* L. Dobrzynski, and B. Djafari-Rouhani \\ Equipe de Dynamique des Interfaces, Laboratoire de Dynamique et Structure des Matériaux Moléculaires, U.R.A. C.N.R.S. No. 801, \\ U.F.R. de Physique Université de LILLE I, 59655 Villeneuve d'Ascq Cédex, France
}

\begin{abstract}
H. Puszkarski
Equipe de Dynamique des Interfaces, Laboratoire de Dynamique et Structure des Matériaux Moléculaires, U.R.A. C.N.R.S. No. 801, U.F.R. de Physique Université de LILLE I, 59655 Villeneuve d'Ascq Cédex, France and Surface Physics Division, Physics Institute, A. Mickiewicz University, Poznan, Matejki 48/49, 60-769 Poland
\end{abstract}

(Received 16 November 1995)

\begin{abstract}
We calculate the spin-wave spectra of two-dimensional composite materials consisting of periodic square arrays of parallel cylinders made of a ferromagnetic material embedded in a ferromagnetic background. Each material is described by its spontaneous magnetization $M_{S}$ and exchange constant $A$. An external static magnetic field is applied along the direction of the cylinders and both ferromagnetic materials are assumed to be magnetized parallel to this magnetic field. We consider the spin-waves propagation in the plane perpendicular to the cylinders. We reveal the existence of gaps in the magnon band structure of composite systems such as the periodic array of Fe cylinders in an EuO matrix. We investigate the existence of these gaps in relation to the physical parameters of the materials involved. We also study the influence of the lattice parameter (i.e., the square array periodicity) and the effect of the filling fraction of the cylinders on the magnon band structure. [S0163-1829(96)09626-9]
\end{abstract}

\section{INTRODUCTION}

In recent years, a large number of papers have addressed the problem of magnon band structure of one-dimensional magnetic composites such as superlattices. Most of these papers focus on the existence of band gaps in the spin waves spectra of magnetic superlattices Albuquerque et al. ${ }^{1}$ calculated the dispersion equation for spin waves propagating in a general direction of an infinite superlattice made of two alternating ferromagnetic layers. They showed that in a certain frequency domain the superlattice dispersion curves exhibit broad pass bands and narrow stop bands. Dobrzynski et al. ${ }^{2}$ investigated the existence of surface-localized magnons in the spin waves spectra of semi-infinite ferromagnetic superlattices. Barnas ${ }^{3}$ analyzed theoretically the spin waves spectra of infinite, semi-infinite, and finite ferromagnetic superlattices in the exchange dominated region. Several authors ${ }^{4,5}$ have carried out the study of magnetic properties of superlattices constructed by alternating films of ferromagnetic and antiferromagnetic layers.

Moreover, during the past decades, the problem of propagation of classical waves in two- and three-dimensional inhomogeneous materials has received a great deal of attention. Of particular interest is the existence of band gaps in the optical, ${ }^{6,7}$ acoustic, ${ }^{8-12}$ or electronic ${ }^{13}$ band structures of periodic as well as random composite media.

On the other hand, recent improvements ${ }^{14,15}$ in the manufacturing of materials permit the fabrication of long $\mathrm{Co}$ or $\mathrm{Cu}$ wires of small diameter (from 50 to $300 \AA$ ). This technology opens the possibility of preparing two-dimensional magnetic composites made of ferromagnetic materials like arrays of long wires in a background.

The above described developments have inspired us to undertake theoretical investigations on this type of two- dimensional inhomogeneous magnetic media. Thus, in this paper, following our previous works on elastic ${ }^{12}$ and electronic $^{13}$ band structures, we present magnon band structure calculations for two-dimensional periodic bimaterial composites such as square arrays of infinite cylinders embedded in a host material.

This paper is organized as follows. In Sec. II, we report briefly the method of calculation of the magnon band structure of two-dimensional periodic media. Spin wave spectra for different composite systems are presented in Sec. III. Finally, some conclusions regarding the propagation of spin waves in inhomogeneous media are drawn in Sec. IV.

\section{METHOD OF CALCULATION}

In this paper, we consider a model system composed of an array of infinite cylinders of circular cross section made of a ferromagnetic material $A$ embedded in an infinite ferromagnetic matrix $B$. The cylinders are assumed to be parallel to the $x_{3}$ axis of the Cartesian coordinate system. Consequently, intersections of their axis with the transverse $\left(x_{1}, x_{2}\right)$ plane form a two-dimensional periodic structure (square lattice; see Fig. 1).

The square lattice constant is $a$ and the filling fractions are $f$ and $(l-f)$ for the materials $A$ and $B$, respectively. In the theoretical model taking the dipolar interactions and the exchange coupling into account, ${ }^{16}$ both ferromagnetic materials $A$ and $B$ are described by their spontaneous magnetization $M_{S_{A}}$ and $M_{S_{B}}$ and exchange constants $A_{A}$ and $A_{B}$. Thus the spontaneous magnetization and the exchange constant in the composite system are space dependent with respect to the position vector $\mathbf{X}=\left(x_{1}, x_{2}\right)$ in the transverse plane and can be written as 

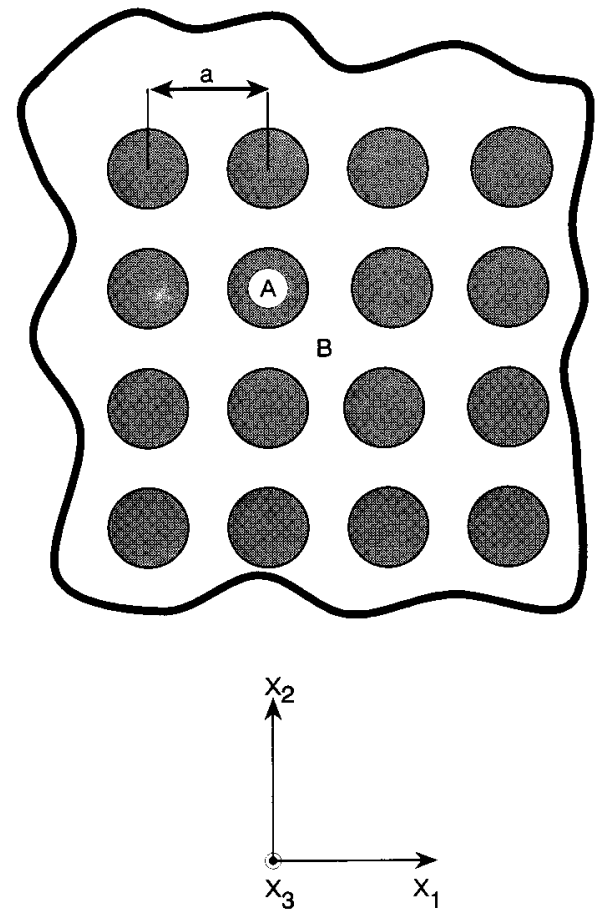

FIG. 1. A transverse cross section of the binary composite system: a square array of infinite cylinders (A) periodically distributed in an infinite matrix (B). The lattice parameter is $a$.

$$
M_{S}=M_{S_{A}} \delta_{\text {in }}+M_{S_{B}}\left(1-\delta_{\text {in }}\right)
$$

and

$$
A=A_{A} \delta_{\text {in }}+A_{B}\left(1-\delta_{\text {in }}\right),
$$

where $\delta_{\text {in }}$ equals 1 inside the inclusions and zero outside. A static magnetic field $\mathbf{H}_{0}$ is applied along the $x_{3}$ axis and both ferromagnetic materials are assumed to be magnetized parallel to $\mathbf{H}_{0}$.

The equation of motion in the composite material is

$$
\frac{\partial}{\partial t} \mathbf{M}(\mathbf{r}, t)=\gamma \mu_{0} \mathbf{M}(\mathbf{r}, t) \times \mathbf{H}_{\mathrm{eff}}(\mathbf{r}, t),
$$

where $\gamma$ is the gyromagnetic ratio $(\gamma<0)$, assumed to be the same in materials $A$ and $B$ and $\mathbf{H}_{\text {eff }}(\mathbf{r}, t)$ is the effective field acting on the magnetization $\mathbf{M}(\mathbf{r}, t)$, $\mathbf{r}$ being the threedimensional position vector. Equation (2) is the LandauLifshitz equation without a damping term. ${ }^{16,17}$

Neglecting an anisotropy field, $\mathbf{H}_{\mathrm{eff}}(\mathbf{r}, t)$ can be written for an inhomogeneous material

$$
\mathbf{H}_{\mathrm{eff}}(\mathbf{r}, t)=H_{0} \mathbf{e}_{3}+\mathbf{h}(\mathbf{r}, t)+\frac{2}{\mu_{0} M_{S}}\left(\nabla \cdot \frac{A}{M_{S}} \nabla\right) \mathbf{M}(\mathbf{r}, t),
$$

where $\nabla=\mathbf{e}_{1}\left(\partial / \partial x_{1}\right)+\mathbf{e}_{2}\left(\partial / \partial x_{2}\right)+\mathbf{e}_{3}\left(\partial / \partial x_{3}\right) \quad\left(\mathbf{e}_{1}, \mathbf{e}_{2}, \mathbf{e}_{3}\right.$ are unit vectors along the $x_{1}, x_{2}, x_{3}$ axis, respectively), $\mathbf{h}(\mathbf{r}, t)$ is the dynamic dipolar field, and the last term describes the exchange field. The difference of this equation from the corresponding one for homogeneous media ${ }^{16}$ is in the $A \cdot M_{S}{ }^{-1}$ scalar put in between the two $\nabla$, in order to satisfy automatically the boundary conditions at the internal interfaces, as done before for photonic, elastic, and electronic composites. ${ }^{6-13}$ In our model the boundary conditions imply the continuity at the internal interfaces of $\left(A / M_{S}\right)(\partial / \partial n) \mathbf{M}$ where $\partial / \partial n$ is an interface normal derivative. Such boundary conditions include the macroscopic variations of $A$ and $M_{S}$ [Eqs. (1)]. They do not take into account the microscopic modifications of the exchange constant $A$ at these interfaces (so-called interface exchange coupling). It is justified to neglect the interface coupling since such microscopic variations of the exchange have negligible effects on the bulk magnons we are going to study in this paper.

In order to resolve Eq. (2), we write

$$
\mathbf{M}(\mathbf{r}, t)=M_{S} \mathbf{e}_{3}+\mathbf{m}(\mathbf{r}, t),
$$

where $\mathbf{m}(\mathbf{r}, t)$ is the dynamic component of the magnetization. We also suppose that

$$
\mathbf{m}(\mathbf{r}, t)=\mathbf{m}(\mathbf{r}) e^{-i \omega t},
$$

where $\omega$ is the wave circular frequency and

$$
\mathbf{h}(\mathbf{r}, t)=\mathbf{h}(\mathbf{r}) e^{-i \omega t},
$$

with $\mathbf{h}(\mathbf{r})=-\nabla \Psi(\mathbf{r})$, where $\Psi(\mathbf{r})$ is a magnetostatic potential. This potential obeys the following equation:

$$
\nabla^{2} \Psi(\mathbf{r})-\lambda\left(\frac{\partial m_{1}(\mathbf{r})}{\partial x_{1}}+\frac{\partial m_{2}(\mathbf{r})}{\partial x_{2}}+\frac{\partial m_{3}(\mathbf{r})}{\partial x_{3}}\right)=0
$$

$[\lambda=4 \pi$ in Gaussian units and $\lambda=1$ in SI (Système International) units used throughout this paper], since

$$
\nabla(\mathbf{h}(\mathbf{r})+\lambda \mathbf{m}(\mathbf{r}))=0 .
$$

We use the usual linear-magnon approximation of neglecting, in Eq. (2), the small terms of second order in $\mathbf{m}(\mathbf{r})$ and $\mathbf{h}(\mathbf{r}){ }^{18}$ This approximation is equivalent to setting $\mathbf{m}(\mathbf{r}) \cdot \mathbf{e}_{3}=0 .^{16}$

On substituting Eqs. (7a), (6), (5), (4), and (3) into Eq. (2), we obtain

$$
\begin{gathered}
i \Omega m_{1}(\mathbf{r})+(\nabla \cdot Q \nabla) m_{2}(\mathbf{r})-m_{2}(\mathbf{r})-\frac{M_{S}}{H_{0}} \frac{\partial \Psi(\mathbf{r})}{\partial x_{2}}=0, \\
-(\nabla \cdot Q \nabla) m_{1}(\mathbf{r})+m_{1}(\mathbf{r})+i \Omega m_{2}(\mathbf{r})+\frac{M_{S}}{H_{0}} \frac{\partial \Psi(\mathbf{r})}{\partial x_{1}}=0,
\end{gathered}
$$

where

$$
\Omega=\frac{\omega}{|\gamma| \mu_{0} H_{0}}=B \omega
$$

and

$$
Q=\frac{2 A}{M_{S} \mu_{0} H_{0}} .
$$

Considering the double periodicity in the $\left(x_{1}, x_{2}\right)$ plane, we can expand $Q$ and $M_{S}$ in Fourier series:

$$
Q(\mathbf{X})=Q\left(x_{1}, x_{2}\right)=\sum_{\mathbf{G}} Q(\mathbf{G}) e^{i \mathbf{G} \cdot \mathbf{X}}
$$




$$
M_{S}(\mathbf{X})=M_{S}\left(x_{1}, x_{2}\right)=\sum_{\mathbf{G}} M_{S}(\mathbf{G}) e^{i \mathbf{G} \cdot \mathbf{X}},
$$

where $\mathbf{G}$ are the reciprocal lattice vectors in the $\left(x_{1}, x_{2}\right)$ plane of components $\left(G_{1}, G_{2}\right)$ and $\mathbf{X}$ is the two-dimensional position vector of components $\left(x_{1}, x_{2}\right)$. The Fourier coefficients in Eq. (9a) take the form

$$
Q(\mathbf{G})=\frac{1}{S} \iint d^{2} \mathbf{X} Q(\mathbf{X}) e^{-i \mathbf{G} \cdot \mathbf{X}},
$$

where the integration is performed over the unit cell surface $S=a^{2}$.

For $\mathbf{G}=0$, Eq. (10) gives the average $Q, \bar{Q}$ :

$$
Q(\mathbf{G}=0)=\bar{Q}=Q_{A} f+Q_{B}(1-f) .
$$

For $\mathbf{G} \neq 0$, Eq. (10) may be written as

$$
Q(\mathbf{G} \neq 0)=\left(Q_{A}-Q_{B}\right) F(\mathbf{G})=\Delta Q F(\mathbf{G}),
$$

where $F(\mathbf{G})$ is the structure factor:

$$
F(\mathbf{G})=\frac{1}{S} \iint d^{2} \mathbf{X} e^{-i \mathbf{G} \cdot \mathbf{X}}=2 f \frac{J_{1}(G R)}{G R},
$$

where $f=\pi\left(R^{2} / a^{2}\right)$ is the filling fraction, $J_{1}$ the Bessel function of the first kind, and $R$ the radius of the cylinders. In Eq. (12), the integration is performed only on material $A$.

In an entirely similar way, Eq. (10) gives

$$
\begin{gathered}
M_{S}(\mathbf{G}=0)=\bar{M}_{S}=M_{S_{A}} f+M_{S_{B}}(1-f), \\
M_{S}(\mathbf{G} \neq 0)=\left(M_{S_{A}}-M_{S_{B}}\right) F(\mathbf{G})=\Delta M_{S} F(\mathbf{G}) .
\end{gathered}
$$

On the other hand, from the property of translational invariance in the $x_{3}$ direction, it follows that $m_{1}(\mathbf{r}), m_{2}(\mathbf{r})$, and $\Psi(\mathbf{r})$ must be of the form

$$
\begin{aligned}
& m_{1}(\mathbf{r})=m_{1}(\mathbf{X}) e^{i K_{3} x_{3}}, \\
& m_{2}(\mathbf{r})=m_{2}(\mathbf{X}) e^{i K_{3} x_{3}}, \\
& \Psi(\mathbf{r})=\Psi(\mathbf{X}) e^{i K_{3} x_{3}},
\end{aligned}
$$

where $K_{3}$ is the $x_{3}$ component of the three-dimensional wave vector $\kappa\left(K_{1}, K_{2}, K_{3}\right)$.

For spin-waves propagation in the $\left(x_{1}, x_{2}\right)$ plane (which means $K_{3}=0$ ), one can consider the two-dimensional wave vector $\mathbf{K}\left(K_{1}, K_{2}\right)$ and use the Bloch theorem to write

$$
\begin{aligned}
& m_{1}(\mathbf{X})=e^{i \mathbf{K} \cdot \mathbf{X}} \sum_{\mathbf{G}} m_{1_{K}}(\mathbf{G}) e^{i \mathbf{G} \cdot \mathbf{X}}, \\
& m_{2}(\mathbf{X})=e^{i \mathbf{K} \cdot \mathbf{X}} \sum_{\mathbf{G}} m_{2_{K}}(\mathbf{G}) e^{i \mathbf{G} \cdot \mathbf{X}}, \\
& \Psi(\mathbf{X})=e^{i \mathbf{K} \cdot \mathbf{X}} \sum_{\mathbf{G}} \Psi_{K}(\mathbf{G}) e^{i \mathbf{G} \cdot \mathbf{X}}
\end{aligned}
$$

One can notice that for $K_{3}=0, \Psi(\mathbf{r})$ does not depend anymore on $x_{3}$. Therefore, $h_{3}(\mathbf{r})=-\left(\partial \Psi(\mathbf{r}) / \partial x_{3}\right)=0$. One observes that for spin-waves propagation in the $\left(x_{1}, x_{2}\right)$ plane and in the linear-magnon approximation, the vectors $\mathbf{m}(\mathbf{r})$ and $\mathbf{h}(\mathbf{r})$ are perpendicular to the $x_{3}$ axis.

After some algebra and considering the dimensionless vectors $\mathbf{k}=(a / 2 \pi) \mathbf{K}$ and $\mathbf{g}=(a / 2 \pi) \mathbf{G}$, the equations of motion can be rewritten as

$$
\begin{aligned}
& i \omega B m_{1_{\mathbf{k}}}(\mathbf{g})=\frac{\lambda \bar{M}_{S}}{H_{0}} \frac{\left(k_{1}+g_{1}\right)\left(k_{2}+g_{2}\right)}{|\mathbf{k}+\mathbf{g}|^{2}} m_{1_{\mathbf{k}}}(\mathbf{g})+\left\{1+\left(\frac{2 \pi}{a}\right)^{2}|\mathbf{k}+\mathbf{g}|^{2} \bar{Q}+\frac{\lambda \bar{M}_{S}}{H_{0}} \frac{\left(k_{2}+g_{2}\right)^{2}}{|\mathbf{k}+\mathbf{g}|^{2}}\right\} m_{2_{\mathbf{k}}}(\mathbf{g})+\sum_{\mathbf{g} \neq \mathbf{g}^{\prime}}\left\{F\left(\frac{2 \pi}{a}\left(\mathbf{g}-\mathbf{g}^{\prime}\right)\right)\right. \\
& \left.\times\left(\frac{\lambda \Delta M_{S}}{H_{0}} \frac{\left(k_{1}+g^{\prime}{ }_{1}\right)\left(k_{2}+g^{\prime}{ }_{2}\right)}{\left(\mathbf{k}+\mathbf{g}^{\prime}\right)^{2}} m_{1_{\mathbf{k}}}\left(\mathbf{g}^{\prime}\right)+\left[\left(\frac{2 \pi}{a}\right)^{2} \Delta Q\left(\mathbf{k}+\mathbf{g}^{\prime}\right)(\mathbf{k}+\mathbf{g})+\frac{\lambda \Delta M_{S}}{H_{0}} \frac{\left(k_{2}+g_{2}^{\prime}\right)^{2}}{\left(\mathbf{k}+\mathbf{g}^{\prime}\right)^{2}}\right] m_{2_{\mathbf{k}}}\left(\mathbf{g}^{\prime}\right)\right)\right\}, \\
& \begin{aligned}
i \omega B m_{2}(\mathbf{g})= & -\left\{1+\left(\frac{2 \pi}{a}\right)^{2}|\mathbf{k}+\mathbf{g}|^{2} \bar{Q}+\frac{\lambda \bar{M}_{S}}{H_{0}} \frac{\left(k_{1}+g_{1}\right)^{2}}{|\mathbf{k}+\mathbf{g}|^{2}}\right\} m_{1_{\mathbf{k}}}(\mathbf{g})-\frac{\lambda \bar{M}_{S}}{H_{0}} \frac{\left(k_{1}+g_{1}\right)\left(k_{2}+g_{2}\right)}{|\mathbf{k}+\mathbf{g}|^{2}} m_{2}(\mathbf{g})-\sum_{\mathbf{k} \neq \mathbf{g}^{\prime}}\left\{F\left(\frac{2 \pi}{a}\left(\mathbf{g}-\mathbf{g}^{\prime}\right)\right)\right. \\
& \left.\times\left(\left[\left(\frac{2 \pi}{a}\right)^{2} \Delta Q\left(\mathbf{k}+\mathbf{g}^{\prime}\right)(\mathbf{k}+\mathbf{g})+\frac{\lambda \Delta M_{S}}{H_{0}} \frac{\left(k_{1}+g_{1}^{\prime}\right)^{2}}{\left(\mathbf{k}+\mathbf{g}^{\prime}\right)^{2}}\right] m_{1_{\mathbf{k}}}\left(\mathbf{g}^{\prime}\right)+\frac{\lambda \Delta M_{S}}{H_{0}} \frac{\left(k_{1}+g_{1}^{\prime}\right)\left(k_{2}+g_{2}^{\prime}\right)}{\left(\mathbf{k}+\mathbf{g}^{\prime}\right)^{2}} m_{2}\left(\mathbf{g}^{\prime}\right)\right)\right\} .
\end{aligned}
\end{aligned}
$$

In going from Eq. (8) to Eqs. (16), $\Psi(\mathbf{r})$ has been eliminated using Eqs. (7a) and (15) with $m_{3}=0$ and $h_{3}=0$.

One notes that there appear in those equations two types of terms: exchange terms depending on $\bar{Q}$ and $\Delta Q$ and dipolar interactions terms depending on $\bar{M}_{s} / H_{0}$ and $\Delta M_{s} / H_{0}$. Equations (16) correspond to an infinite set of linear equations where the unknowns are the Fourier components of the magnetization. In practice, obviously, only a finite number of $\mathbf{g}$ vectors are taken into account for the numerical calculation. The determinant of this system of equations must vanish, which conditions yield the magnon band structure $\omega_{n}(\mathbf{k})$. Despite the fact that the equations (16) involve complex imaginary terms, their solutions $\omega_{n}(\mathbf{k})$ are real. One can also notice that in Eqs. (16) there is an explicit dependence of the frequency $\omega$ with the lattice parameter $a$. In the case of photonic ${ }^{6}$ and phononic band structures ${ }^{8-12}$ such a dependence is implicit in the sense that the band structures are given in terms of a reduced frequency depending on the lattice parameter $(\Omega=\omega a / 2 \pi c$ where $c$ is a velocity) versus the reduced wave vector. Regarding Eqs. (16), 
TABLE I. Values of the exchange constant $A$ and spontaneous magnetization $M_{S}$ for $\mathrm{Fe}$ (Refs. 16 and 17), $\mathrm{EuO}$ (Refs. 17, 20, and 21), Co (Ref. 16), and Permalloy (Ref. 16).

\begin{tabular}{lcc}
\hline \hline & $\begin{array}{c}A \\
\left(10^{-11} \mathrm{~J} \mathrm{~m}^{-1}\right)\end{array}$ & $\begin{array}{c}M_{S} \\
\left(10^{6} \mathrm{~A} \mathrm{~m}^{-1}\right)\end{array}$ \\
\hline $\mathrm{Fe}$ & 2.1 & 1.752 \\
$\mathrm{EuO}$ & 0.1 & 1.910 \\
$\mathrm{Co}$ & 2.8 & 1.390 \\
Permalloy & 0.7 & 0.810 \\
\hline \hline
\end{tabular}

one can observe that, due to the existence of the dipolar interaction terms, it is not enough to define a reduced frequency to take fully into account the effect of the lattice parameter $a$ on the magnon band structure.

\section{MAGNON BAND STRUCTURES}

In this section, we present magnon band structures calculated for square arrays of Fe cylinders in a EuO background as well as square array of Co cylinders in a Permalloy matrix. The inverse situations, i.e., EuO cylinders in an Fe matrix, and Permalloy fibers in a Co matrix have also been investigated. Fe, EuO, Permalloy, and Co are ferromagnetic materials. Their physical parameters $M_{S}$ and $A$ are listed in Table I.

In the case of the two-dimensional periodic system, $\mathrm{Fe}$ (cylinders)/EuO (background), the influence of the Fe filling fraction and the effect of the lattice parameter on the band structure are also studied. In the course of the numerical

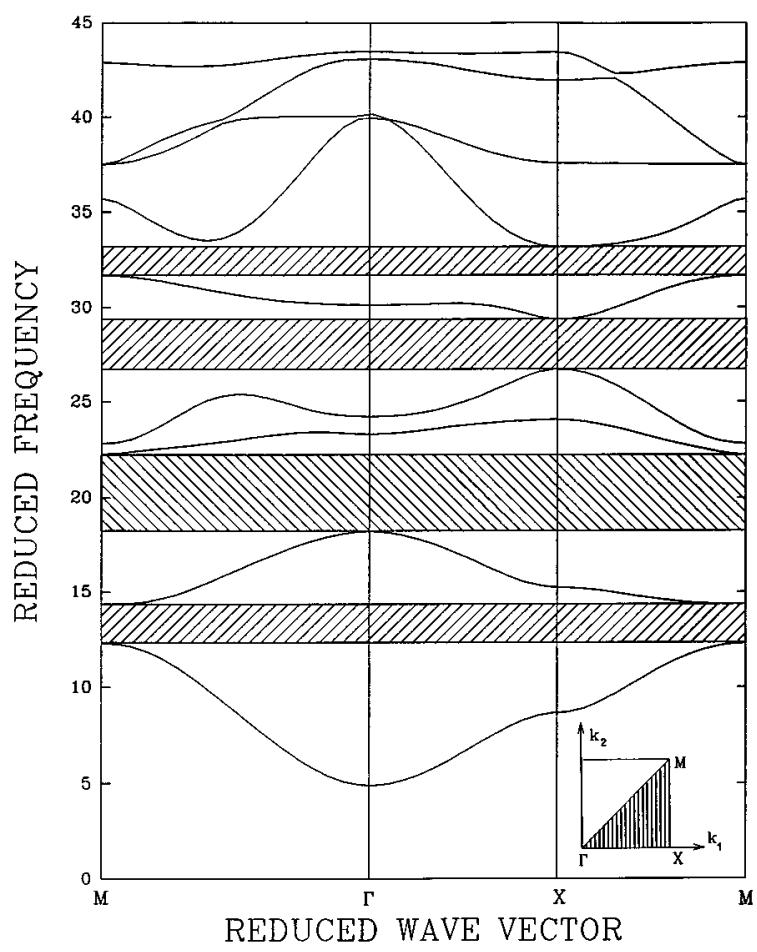

FIG. 2. Magnon band structure for Fe cylinders in a EuO matrix for a filling fraction $f=0.5, a=100 \AA$, and $\mu_{0} H_{0}=0.1 \mathrm{~T}$. The band structure is plotted in the three high-symmetry directions ГХM of the Brillouin zone (see inset). One can notice four gaps.

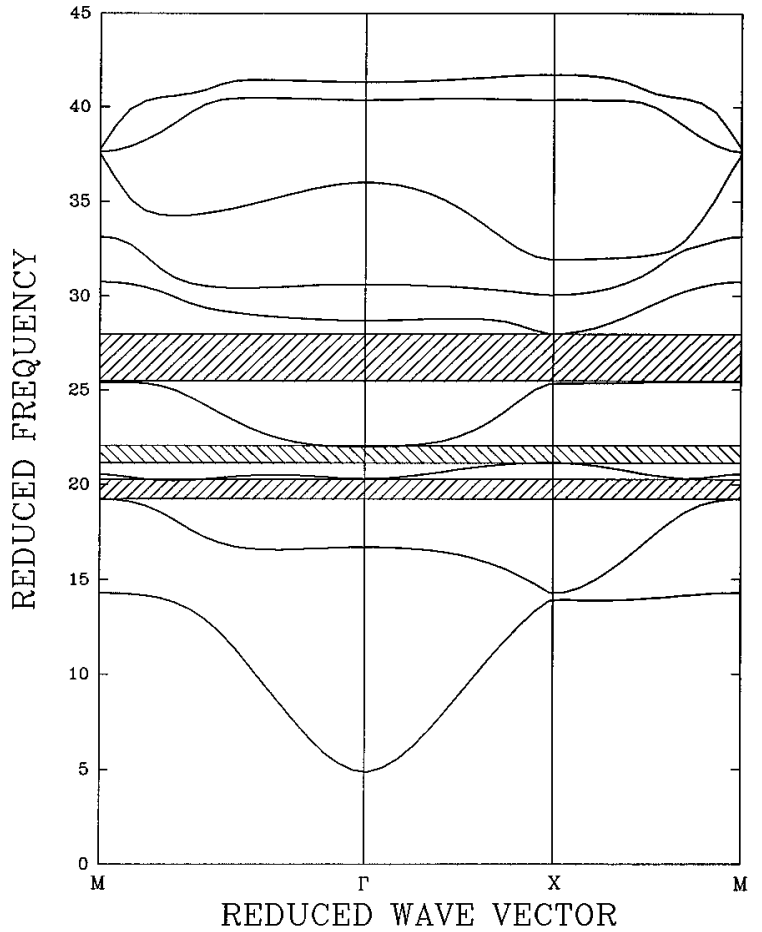

FIG. 3. Magnon band structure for EuO cylinders in an Fe matrix for a filling fraction $f=0.5, a=100 \AA$, and $\mu_{0} H_{0}=0.1 \mathrm{~T}$. There are three gaps. The width of the first two gaps is lower compared to Fig. 2. The width of the third gap is of the same order of magnitude in both cases.

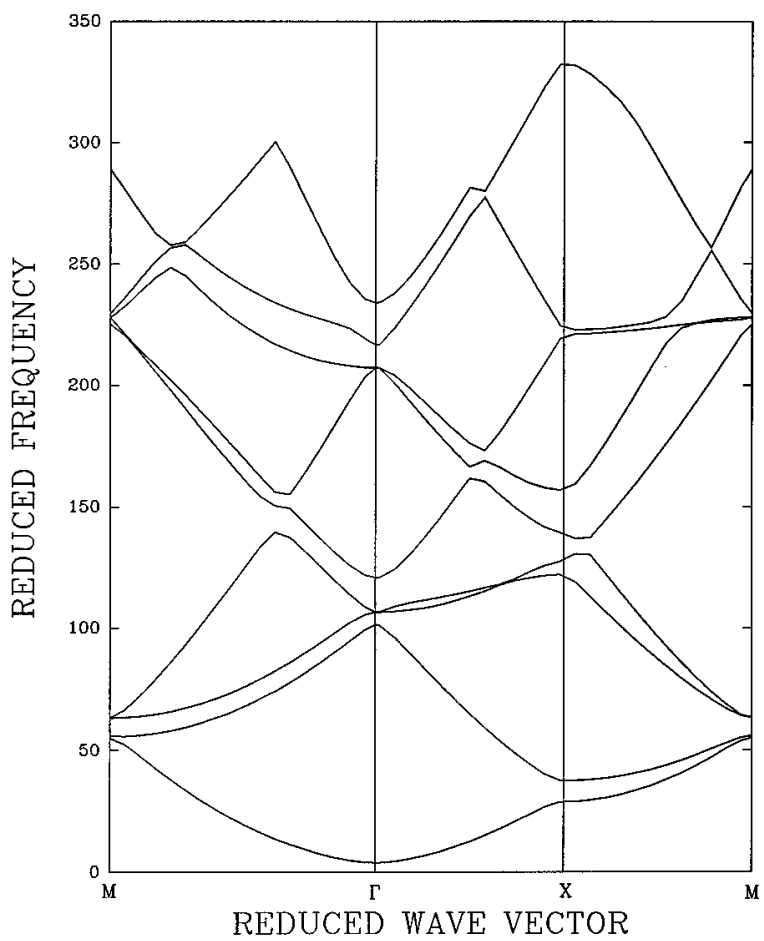

FIG. 4. Magnon band structure for Co cylinders in a Permalloy matrix for a filling fraction $f=0.5, a=100 \AA$, and $\mu_{0} H_{0}=0.1 \mathrm{~T}$. There is no gap in this case either in the opposite situation (i.e., Permalloy cylinders in a Co matrix). 


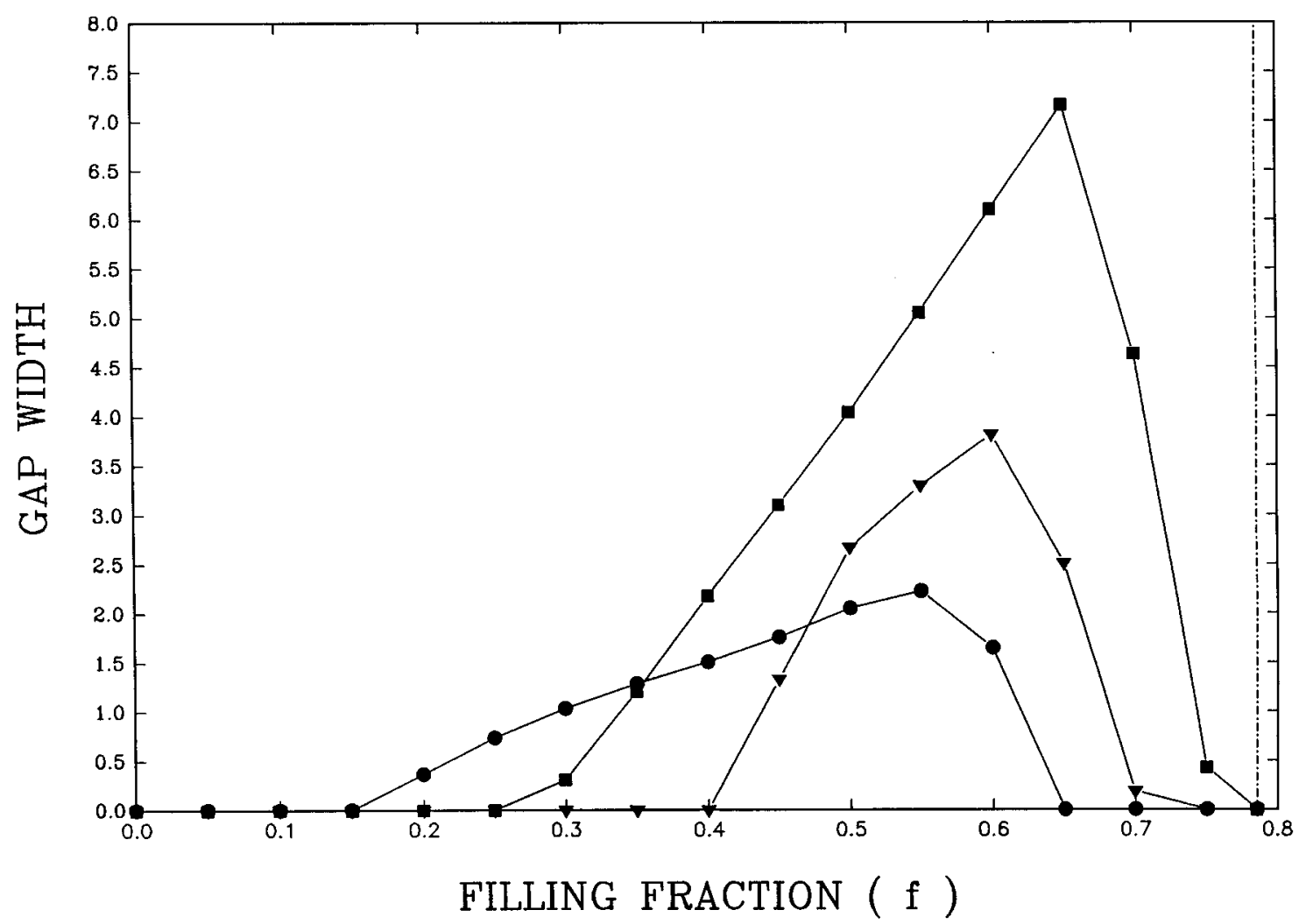

FIG. 5. The width of the first three band gaps in the magnon band structure of the square array of Fe cylinders in a EuO matrix for $a=100$ $\AA$ and $\mu_{0} H_{0}=0.1 \mathrm{~T}$ as a function of the filling fraction $f$. The vertical dashed line corresponds to the close-packing value of $f(f=\pi / 4)$ for which one cylinder contacts another one. Filled circles: first gap (between the first and the second band). Filled squares: second gap (between the second and the third band). Filled triangles: third gap (between the fourth and the fifth band).

calculations, the dimensionless reciprocal lattice vectors $\mathbf{g}$ are given as $\mathbf{g}=n_{1} \mathbf{e}_{1}+n_{2} \mathbf{e}_{2}$, where $n_{1}$ and $n_{2}$ are two integers limited to the interval $-N \leqslant n_{1}, n_{2} \leqslant+N$. All the results sketched below are obtained with $N=6$. However, some of the dispersion curves were also calculated with $N=10$ and confirmed the good accuracy of the results for $N=6$. The difference in the eigenvalues calculated with $N=10$ and 6 is small. We choose $N=6$ which is a good compromise between accuracy and computing time.

Figure 2 shows the first nine magnon bands for the square array of $\mathrm{Fe}$ cylinders in a $\mathrm{EuO}$ matrix, the filling fraction $f$ being equal to 0.5 . We have plotted the magnon band structure in the three principal directions of the first twodimensional Brillouin zone ГXM (see the inset in Fig. 2). The plots are given in terms of the reduced frequency $\Omega=B \omega=\omega /|\gamma| \mu_{0} H_{0}$, versus the reduced Bloch wave vector $\mathbf{k}$. The reduced frequency $\Omega$ is defined here as being independent of the lattice parameter $a$. The lattice parameter $a$ is equal to $100 \AA$ (the radius $R$ of the cylinders is then equal to $40 \AA$ ) and $\mu_{0} H_{0}=0.1 \mathrm{~T}$.

In the range of frequency of Fig. 2, four band gaps appear, respectively, between the first and the second band, between the second and the third band, between the fourth and the fifth band, and between the fifth and the sixth band. The widths of the first two gaps are of the order of 2 and 4 reduced units, respectively, which correspond to frequencies of the order of 5.5 and $11 \mathrm{GHz}$.

Figure 3 shows the magnon band structure in the inverse situation, i.e., the square array of EuO cylinders in a Fe matrix. In this case, the magnitudes of the first two gaps (close to $2 \mathrm{GHz}$ ) are smaller than those obtained in the previous case. However, the width of the third gap (appearing between the fourth and the fifth band) is of the same order of magnitude as in the former case.

In Fig. 4, the magnon band structure for a square array of Co cylinders in a Permalloy matrix is drawn for $f=0.5$. The other parameters used are the same as in Figs. 2 and 3. There is no gap in this case, neither in the inverse situation (Permalloy cylinders in a Co matrix). One can notice that for the two binary composite systems Fe (cylinders)/EuO (matrix) and Co (fibers)/Permalloy (background), the inclusions and the matrix have more or less the same spontaneous magnetization but different exchange constants (see Table I).

We also considered a hypothetic binary composite system where the cylinders and the matrix have the same exchange constant but different spontaneous magnetizations. In that case, gaps appear in the band structure only for the ratio $M_{S_{A}} M_{S_{B}}{ }^{-1}$ greater than 10 or lower than 0.1 , i.e., for a big difference in spontaneous magnetizations. Most of the usual ferromagnetic materials ${ }^{17}$ (Fe, Co, Ni, Gd, EuO, Permalloy) have spontaneous magnetizations of the same order of magnitude (from $0.5 \times 10^{6}$ to $2 \times 10^{6} \mathrm{~A} \mathrm{~m}^{-1}$ ).

From these results, one can think that the existence of large gaps in the magnon band structure of square arrays of ferromagnetic cylinders embedded in a ferromagnetic back- 


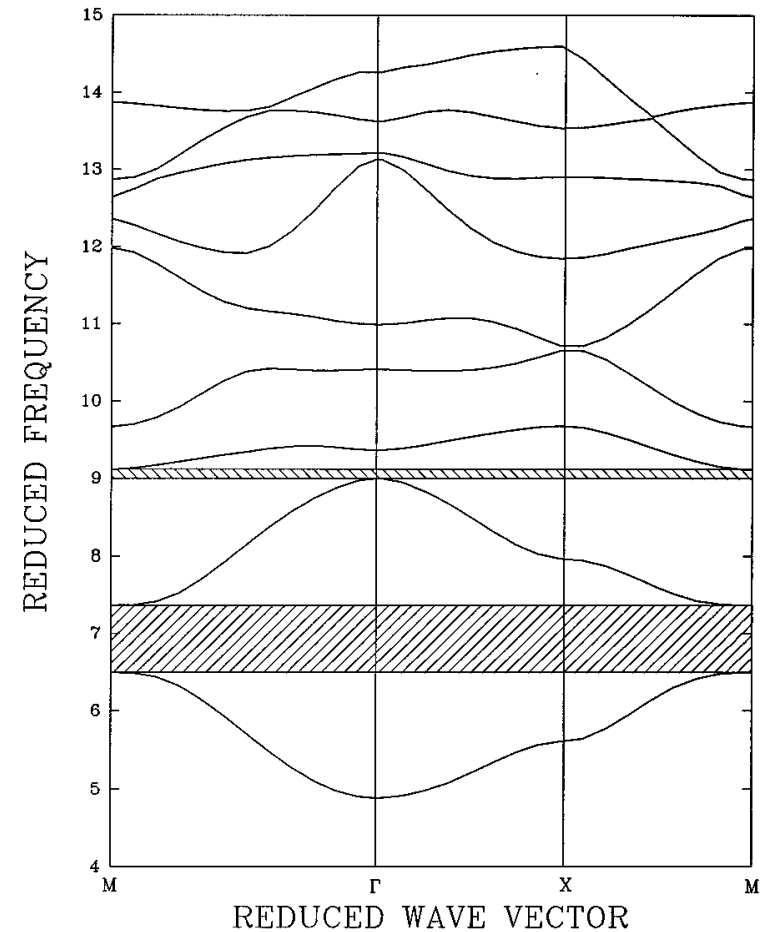

FIG. 6. The same as in Fig. 2 for $a=250 \AA$.

ground is associated with a very strong exchange contrast. The choice of an inclusion component of greater exchange constant than the matrix is more favorable for the opening of gaps in the magnon band structure. In Co and Permalloy, the exchange constants are of the same order of magnitude whereas the $\mathrm{Fe}$ exchange constant is twenty-one times greater than the one of EuO. One can also notice that the necessity of a strong contrast between the physical parameters of the inclusions and the matrix has already been observed in our previous work on elastic waves ${ }^{12}$ and electrons. ${ }^{13}$

We also studied the influence of the inclusion filling fraction on the magnon band structure of two-dimensional periodic ferromagnetic systems. In Fig. 5, the widths of the first three gaps in the magnon band structure of the square array of $\mathrm{Fe}$ cylinders in an $\mathrm{EuO}$ background are given as a function of the inclusion filling fraction $f$ for $a=100 \AA$. We note the opening of gaps over a large range of the filling fraction, namely, $0.15<f<0.75$. The maximum gap width is obtained for $f=0.65$. However, only two gaps appear for this value of $f$.

We also investigated the effect of the lattice parameter on the magnon band structures. First of all, one can notice, looking at the equations of motion [Eqs. (16)], that the reduced frequency $\Omega$ depends on exchange terms and dipolar interactions terms, exchange terms being multiplied by the factor $(2 \pi / a)^{2}$. As a result, for very low $a$, the magnon frequencies are not affected by the dipolar interactions. In contrast, for very large $a$, the first few magnon bands presented in our illustrations are strongly affected by the dipolar interactions. In Figs. 6 and 7, the magnon band structures for the square array of $\mathrm{Fe}$ cylinders in a EuO matrix are plotted for two different values of the lattice parameter, $a=250$ and $500 \AA$,

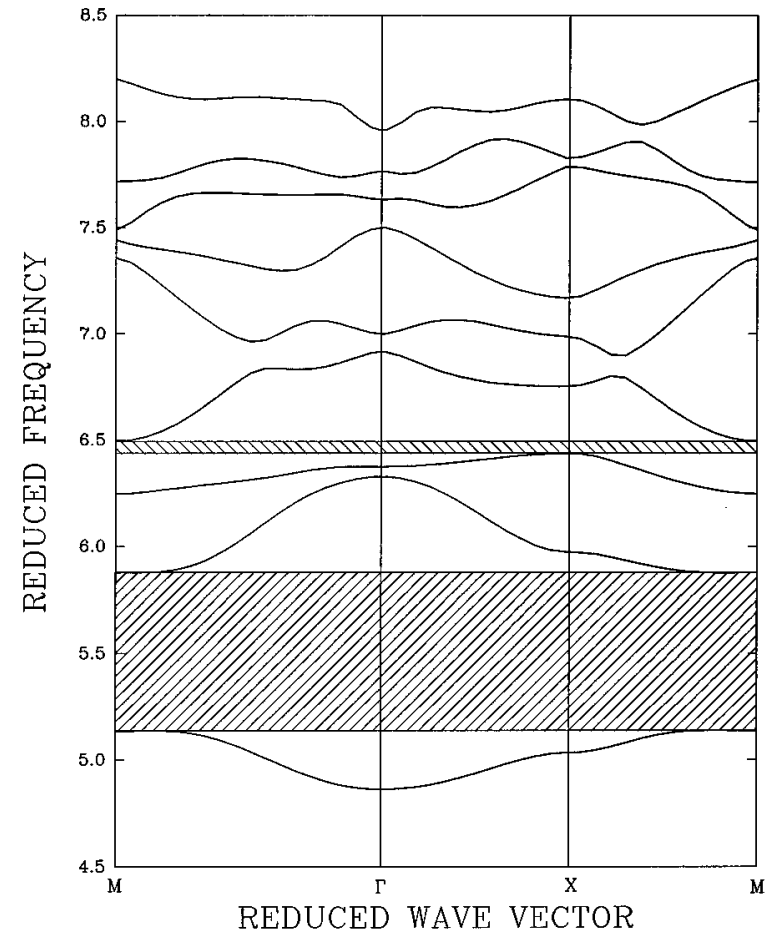

FIG. 7. The same as in Fig. 2 for $a=500 \AA$.

respectively. Comparing Figs. 2, 6, and 7, one observes that the bottom of the first band ( $\Gamma$ point) still appears at, approximately, the same reduced frequency $(\Omega \approx 4.9)$. On the other hand, the first gap width (as well as its location on the frequency scale) decreases with increasing lattice parameter. Moreover, on Figs. 6 and 7, the gaps of upper frequency domains (see Fig. 2) are very narrow and some have disappeared.

The opening of gaps in the magnon band structure of periodic square arrays of ferromagnetic cylinders embedded in a ferromagnetic matrix appears to be favored for arrays of low lattice parameter $(a \approx 100 \AA)$. One can notice that for $f=0.5$ and $a=100 \AA$, the radius of the cylinders is $R=40 \AA$. This value corresponds approximately to the lower limit of the radius of the $\mathrm{Co}$ and $\mathrm{Cu}$ cylinders manufactured experimentally. ${ }^{14,15}$

\section{CONCLUSIONS}

The purpose of this paper was to investigate theoretically the existence of band gaps in the magnon band structures of two-dimensional composite systems composed of periodic arrays of infinite ferromagnetic cylinders embedded in a ferromagnetic matrix. For the periodic systems, $\mathrm{Fe}$ (cylinders)/ $\mathrm{EuO}$ (matrix) and $\mathrm{EuO}$ (cylinders)/Fe (matrix), we obtained absolute band gaps for which the spin wave propagation in directions perpendicular to the cylinders axis is forbidden. We have found that the gaps widths are in the range of 2-11 $\mathrm{GHz}$, which is well above the experimentally available frequency resolution. ${ }^{17,19}$ The influence of the inclusion filling fraction and the effect of the lattice parameter on the band structure were also studied. The existence of a strong con- 
trast between the exchange constants of the inclusions and the matrix appears to be a necessary condition to obtain gaps in the magnon band structure of ferromagnetic composite systems. The width of these gaps and their frequency domains are strongly affected by the composition of the composite system and by the lattice parameter. It could be interesting to extend our calculations to other geometries of the composite system and to other fiber cross sections.

\section{ACKNOWLEDGMENTS}

One of the authors (H.P.) wishes to express his gratitude to the Laboratoire de Dynamique et Structure des Matériaux Moléculaires, Unité de Recherche Associée au C.N.R.S. No. 801, Université de Lille I, for their hospitality and to Le Conseil Régional (Nord-Pas de Calais) for its support; thanks are also due to the Polish Committee for Scientific Research for support under the Grant No. 2 Po3B 04308.
*Author to whom correspondence should be addressed.

${ }^{1}$ E. L. Albuquerque, P. Fulco, E. F. Sarmento, and D. R. Tilley, Solid State Commun. 58, 41 (1986).

${ }^{2}$ L. Dobrzynski, B. Djafari-Rouhani, and H. Puszkarski, Phys. Rev. B 33, 3251 (1986).

${ }^{3}$ J. Barnas, Phys. Rev. B 45, 10427 (1992).

${ }^{4}$ L. L. Hinchey and D. J. Mills, Phys. Rev. B 33, 3329 (1986).

${ }^{5}$ L. L. Hinchey and D. J. Mills, Phys. Rev. B 34, 1689 (1986).

${ }^{6}$ M. Plihal and A. A. Maradudin, Phys. Rev. B 44, 8565 (1991) and references therein.

${ }^{7}$ E. Yablonovitch, J. Opt. Soc. Am. B 10, 283 (1993) and references therein.

${ }^{8}$ M. M. Sigalas and E. N. Economou, J. Sound Vib. 158, 377 (1992).

${ }^{9}$ M. M. Sigalas and E. N. Economou, Solid State Commun. 86, 141 (1993).

${ }^{10}$ M. S. Kushwaha, P. Halevi, L. Dobrzynski, and B. DjafariRouhani, Phys. Rev. Lett. 71, 2022 (1993).

${ }^{11}$ M. S. Kushwaha, P. Halevi, G. Martinez-Montes, L. Dobrzynski, and B. Djafari-Rouhani, Phys. Rev. B 49, 2313 (1994).
${ }^{12}$ J. O. Vasseur, B. Djafari-Rouhani, L. Dobrzynski, M. S. Kushwaha, and P. Halevi, J. Phys. Condens. Matter 6, 8759 (1994).

${ }^{13}$ L. Dobrzynski, B. Djafari-Rouhani, J. Vasseur, R. Kucharczyk, and M. Steslicka, Prog. Surf. Sci. 48, 213 (1995).

${ }^{14}$ A. Blondel, J. P. Meier, B. Doudin, and J. Ph. Ansermet, Appl. Phys. Lett. 65, 3019 (1994).

${ }^{15}$ J. de la Figuera, M. A. Huerta-Garnica, J. E. Prieto, C. Ocal, and R. Miranda, Appl. Phys. Lett. 66, 1006 (1995).

${ }^{16}$ M. Vohl, J. Barnas, and P. Grünberg, Phys. Rev. B 39, 12003 (1989).

${ }^{17}$ F. Keffer, Spin Waves, Handbuch der Physik Vol. XVIII/2 (Springer, Berlin, 1966).

${ }^{18}$ M. G. Cottam and D. R. Tilley, Introduction to Surface and Superlattice Excitations (Cambridge University Press, Cambridge, England, 1989).

${ }^{19}$ M. G. Cottam and O. J. Lockwood, Light Scattering in Magnetic Solids (Wiley, New York, 1987).

${ }^{20}$ B. T. Matthias, R. M. Bozorth, and J. H. Van Vleck, Phys. Rev. Lett. 7, 160 (1961).

${ }^{21}$ J. F. Dillon and C. E. Olsen, Phys. Rev. 135, A434 (1964). 